\title{
Numerical study of the influence of interfacial roughness on the exchange bias properties of ferromagnetic/antiferromagnetic bilayers
}

\author{
J. Moritz* \\ Institut Jean Lamour, UMR 7198 CNRS - Université de Lorraine, Bd des Aiguillettes, BP 70239 , \\ F-54506 Vandoeuvre-les-Nancy Cedex, France \\ P. Bacher \\ ESSTIN - Université de Lorraine - 2, rue Jean Lamour, F-54519 Vandoeuvre-les-Nancy Cedex, France
}

\author{
B. Dieny \\ Université Grenoble Alpes, INAC-SPINTEC, F-38000 Grenoble, France; \\ CEA, INAC-SPINTEC, F-38000 Grenoble, France \\ and CNRS, SPINTEC, F-38000 Grenoble, France
}

(Received 25 April 2016; revised manuscript received 28 July 2016; published 22 September 2016)

\begin{abstract}
Exchange bias and coercivity are both studied numerically in antiferromagnetic/ferromagnetic (AFM/FM) bilayers in the presence of a rough interface. The roughness is modeled by an AFM atomic mesa of variable width, in a periodic bidimensional system. Unlike the flat interface case, roughness can favor the presence of magnetic interfacial frustration or the formation of sharp magnetic domain walls pinned within the first AFM planes, inside the AFM mesa, in a Peierls potential well. We demonstrate by using athermal steepest-descent calculations that irreversible processes can occur during the hysteresis loops, when the AFM mesa width is less than half of the system period. In this case, the depinning of the domain wall from the Peierls potential well during the descending branch is not followed by its rewinding in a certain range of the AFM anisotropy. This leads to a large increase of both exchange bias and coercivity at low temperature and to an athermal training effect. When the thermal activation is taken into account by using Monte Carlo simulations, we show that a random walk of the domain wall occurs within the AFM layer. These processes induce changes in the AFM spin configuration when the system is cycled several times and produce a thermally activated training effect. Our simulations, interpreted in the context of periodic Peierls potential, provide an explanation for two important features of the exchange bias phenomenon, i.e., the thermal variation of its characteristic fields and the different contributions giving rise to the training effect (AFM bulk vs interface). More generally, the presence of interfacial atomic roughness reduces both exchange bias and coercivity with respect to the perfect interface case.
\end{abstract}

DOI: 10.1103/PhysRevB.94.104425

\section{INTRODUCTION}

Exchange coupled ferromagnetic/antiferromagnetic (FM/AFM) bilayers are extensively used in spintronic devices (magnetic read-heads, magnetic RAMs, etc.) to establish a reference magnetization direction [1]. This is achieved by cooling down the FM/AFM bilayer under an applied magnetic field from above the Néel temperature of the AFM film (or at least above its blocking temperature). The resulting hysteresis loop of the FM layer measured at a temperature much below the AFM blocking temperature exhibits a shift along the applied field axis. The larger is the field shift, called the exchange bias field $\left(H_{E X}\right)$, the better is the stability of the reference layer. When the loop shift exceeds the coercive field $\left(H_{C}\right)$, only one remanent state is stable at zero field, a situation of particular interest for spintronic devices.

This interfacial effect, discovered more than 60 years ago [2], has been studied in many different magnetic FM/AFM systems, epitaxial or polycrystalline, either in continuous films or in the form of patterned magnetic nanostructures (see Refs. [3-6] for topical reviews and recent results on patterned systems [7-9]). With the first and simplest model

\footnotetext{
*jerome.moritz@univ-lorraine.fr
}

of exchange anisotropy [10], based on perfect uncompensated (all spin-aligned) FM/AFM interface, $H_{E X}$ was expected to be proportional to the interface exchange coupling $J_{0}$. Unfortunately, this simple picture yielded an exchange bias energy two orders of magnitude larger than the one measured experimentally. In order to theoretically derive a correct order of magnitude of the loop shift along the field axis, a deeper understanding of the magnetic properties of the FM/AFM interface was necessary.

A first proposed reason for the decrease of the interfacial energy is the presence of roughness at the FM/AFM interface. This can lead to the formation of magnetic domains in the AFM layer. In such a case, $H_{E X}$ is found to be of the order of the domain-wall energy $\sqrt{J K}$ expressed in energy units, where $J$ is the magnetic exchange energy between AFM atoms and $K$ is their anisotropy energy [11]. Assuming $J_{0} \simeq J$, the loop shift is reduced by a factor $\delta=\sqrt{J / K}$ within this approach, with $\delta$ being the dimensionless domain-wall (DW) width expressed in atomic distance units. Impurities in the AFM bulk can also induce a breaking up of the antiferromagnet into domains of different orientations, leading to a drastic reduction of $H_{E X}$ with respect to $J_{0}$ [12]. In polycrystalline layers though, if no intergrain couplings are considered, each AFM grain can be considered as being single domain. The AFM grain stability during hysteresis loops depends on its volume and aspect ratio, 
the characteristic experimental time, temperature, and different energy terms $\left(J_{0}, K\right)$ : its staggered magnetization can remain pinned in its original setting direction and contribute to the exchange bias, or switch in the opposite direction and increase the coercivity of the bilayer [13-15].

Concerning the enhanced coercivity which is experimentally observed in AFM/FM bilayers (with respect to the identical single FM layer), it is usually ascribed to irreversible changes occurring within the AFM layer, such as grain reversal, partial DW reversal within single crystallites [16,17], AFM DW depinning from local defects [11,12], spin-flop coupling [18], or interface magnetic frustration [19]. Therefore, $H_{E X}$ and $H_{C}$ are expected to be connected in some way because they are both related to the magnetic configuration changes in the AFM layer.

As a matter of fact, the magnetic configuration of the antiferromagnet depends on the magnetic history of the sample, such as the number of hysteresis loops, previous thermal explorations, and field and temperature setting conditions to which the bilayers have been exposed [14,20]. For instance, when an exchange biased FM layer is cycled $n$ times, a decrease of $H_{E X}$ (and $H_{C}$ ) vs $n$ is observed in a wide variety of FM/AFM systems. This effect, evidenced by Paccard 50 years ago [21], is usually ascribed to irreversible domain rearrangements at the FM/AFM interface and to a decrease of the net interfacial AFM moment during the successive hysteresis loops [22]. A phenomenological law in $\sqrt{n}$, or in power of $n$, is generally used to fit the data, although the experimental decrease of the hysteresis between the first and the second loop is often too large with respect to the expected one [23,24]. Several authors claimed the symmetry of the AFM anisotropy (uniaxial vs biaxial) is responsible for the training observed between the first and second hysteresis loops (athermal training), whereas classical thermal activation causes the training during the subsequent loops [25-27]. Granular models, taking into account intergrain coupling, were also used to explain the origin of the athermal training [28], although some authors argued that only interfacial effects are involved in this phenomenon [29].

We recently extended the Néel-Mauri model of the formation of a planar DW parallel to the FM/AFM interface by introducing a source of intrinsic DW pinning [30,31]. In this model, the interfacial exchange energy cost associated with the FM magnetization reversal is minimized by the formation of a DW parallel to the interface within the AFM film. In the original Néel-Mauri model, the formation/annihilation of this DW was assumed to be reversible, i.e., the energy given to the system at the DW creation during the descending hysteresis branch is completely released during the ascending branch, leading to exchange bias and no coercivity. We brought a additional ingredient to the formulation of Néel-Mauri by introducing the pseudoperiodic Peierls potential in which the DW moves within the AFM crystal lattice [32]. In a highanisotropy system (AFM thin films exhibit usually crystalline anisotropy [33-35] within the range $10^{6}-10^{7} \mathrm{~J} / \mathrm{m}^{3}$ ), the DWs are very sharp and extend over a few interatomic distances only. Making the DW propagate throughout the magnetic layer requires its central spin to be brought periodically perpendicular to the easy axis, where the total magnetic energy is maximum [36-39]. This Peierls potential energy is often considered to be responsible for the large coercivity measured in hard permanent magnets at low temperature [40,41].

We showed that in FM/AFM bilayers, similar intrinsic pinning arises for DWs parallel to the interface within the AFM films. During the descending branch of the hysteresis loop, the first critical field is associated with the Zeeman energy needed to create the DW, i.e., $H_{1}=-\sqrt{J K}$ in energy units. On the other hand, during the ascending branch, the unwinding of the DW is not a reversible process because the Peierls potential has to be overcome, so that $H_{2}=H_{1}+H_{d}$, where $H_{d}$ is the intrinsic depinning field. Two distinct anisotropy thresholds were then evidenced:

(i) For a given AFM thickness $t_{A F M}$, the anisotropy should be large enough in order to have $\delta \simeq t_{A F M}$, otherwise the Peierls potential well is not deep enough to pin the partial DW. If this condition is not fulfilled, this results in the entire reversal of the AFM spins, i.e., large $H_{C}$, no $H_{E X}$.

(ii) When $\sqrt{J K}>J_{0}$, the formation of the DW is not the most favorable energetic process, so that $H_{1}=H_{2}=-2 J_{0}$, i.e., $H_{E X}=-2 J_{0}, H_{C}=0$.

The effect of the temperature was also studied [42]. We showed that sharp DWs can move according to a random walk within the AFM layer, jumping from one minimum of the pseudoperiodic Peierls potential to another. If the temperature is high enough or the experimental time sufficiently long, the DW can be expelled out from the AFM layer, leading to the entire reversal of the AFM spins. This induces a reduction of both $H_{E X}$ and $H_{C}$ versus temperature, as well as a magnetic after-effect behavior similar to experiments [27,43].

Our previous studies were considering the case of perfect FM/AFM interfaces. It is well known though that roughness can induce magnetic frustration at the interface or can lead to the pinning of sharp DW within the first AFM planes in the setting conditions. In this paper, we investigate by numerical simulations the effect of interfacial roughness on the exchange bias and coercivity.

In a first part, we describe the studied system, the setting conditions, and the numerical procedure. In a second part, the calculated athermal variations of both $H_{E X}$ and $H_{C}$ are presented as a function of the $K / J$ ratio for different initial configurations of the system. The results are interpreted in the context of the Peierls intrinsic pinning. Finally, in a third part, we show how the temperature changes the reversal mode of the AFM layer, in this case of rough interface. Thermal variations of both $H_{E X}$ and $H_{C}$, as well as their training effect, exhibit a relative good agreement with experimental data.

\section{MODEL}

We consider in this paper a two-dimensional (2D) noncompensated AFM layer with a rough interface coupled to a FM one with a lattice parameter $a=1$. The system is composed of a total of eight atomic planes, labeled from $i=-1$ to $i=6$, and is periodic in the $x$ direction (see Fig. 1). The FM/AFM interface is located at the plane $i=1$, and is composed of both AFM and FM atoms, while the planes $i=-1,0$ and $i$ from 2 to 6 are made of single FM and AFM atoms, respectively. In order to vary the proportion of AFM vs FM atoms at the interface, two column of spins are considered, labeled $j=0$ and $j=1$. The width of the periodic studied cell in the $(O x)$ 

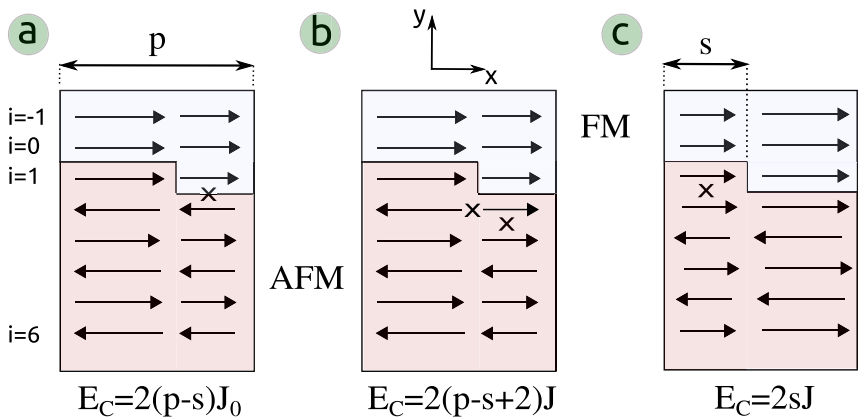

FIG. 1. Sketch of the FM/AFM studied bilayer. Two columns of spins of eight atomic planes each are considered, with five AFM and two FM planes being completely filled. In order to describe an atomic mesa of width $s$ at the rough interface, the spins within the left column are weighted by $s$ and by $p-s$ within the right column, with $p$ the system periodicity in the $x$ direction. The three configurations (a) $a$, (b) $b$, and (c) $c$ are the possible initial states of the system, whose respective energies $E_{C}$ are given in the figure.

direction is $p=10$. The first (left) column $j=0$ is weighted by $s$ and the second one $j=1$ by $p-s$, so that there is an AFM mesa of width $s$ at the interface, as depicted in Fig. 1. This way of weighting each spin column allows the change of the AFM atoms' proportion at the interface, between $s=1$ to $s=9$, for the chosen periodicity $p=10$.

The AFM layer exhibits a uniaxial anisotropy $K$, whereas the FM film is a soft magnetic material without magnetocrystalline anisotropy. Each FM spin carries a magnetization $M=1$, and the total magnetic moment is $M_{s}=20+p-s$, which is the sum of the moments of two full FM planes $(2 \times p)$, added to the FM atoms moment at the interface within the column $j=1$ of width $p-s$.

The exchange constant at the interface between FM and AFM atoms is $J_{0}=0.7, J_{F}=0.9$ within the FM layer and $J=-0.5$ in the AFM bulk. As a simplified way to take into account the demagnetizing energy, we assume that each FM spin is free to rotate in the $(O x z)$ plane only, with $\theta_{i, j}$ being the angle of the spins with respect to the $(O x)$ AFM easy-axis direction. As we mainly focus in this study on the effect of the torque exerted by the FM magnetization at the interface on the stability of the AFM layer, we also neglect the coupling between the external field $H$ and the AFM spins.

Under these last assumptions, the total magnetic energy of the system is the sum of exchange, anisotropy, and Zeeman energy terms. The exchange energy between two neighboring spins is of the form $2 J(1-\cos \delta \theta)$, with $J$ the exchange constant (that could be positive or negative depending on the interaction type) and $\delta \theta$ the angle between both spins. As a result, the total exchange energy $E_{F}$ between FM atoms is

$$
\begin{aligned}
E_{F}= & s \times 2 J_{F}\left[1-\cos \left(\theta_{0,0}-\theta_{-1,0}\right)\right]+(p-s) \\
& \times 2 J_{F} \sum_{i=-1,0}\left[1-\cos \left(\theta_{i+1,1}-\theta_{i, 1}\right)\right] \\
& +2 \times 2 J_{F} \sum_{i=-1,0}\left[1-\cos \left(\theta_{i, 1}-\theta_{i, 0}\right)\right] .
\end{aligned}
$$

The first term is the exchange interaction between FM spins in the $(O y)$ direction within the first column weighted by $s$; the second one is the same kind of interaction but within the second column weighted by $p-s$. The last term represents the exchange interaction in the $(O x)$ direction, which is counted double because of the periodicity of the system. The total exchange energy between AFM atoms $E_{A F}$ and at the interface between AFM and FM atoms $E_{I N T}$ can be similarly written:

$$
\begin{aligned}
E_{A F}= & \times 2 J \sum_{i=1}^{5}\left[1-\cos \left(\theta_{i+1,0}-\theta_{i, 0}\right)\right]+(p-s) \\
\times & 2 J \sum_{i=2}^{5}\left[1-\cos \left(\theta_{i+1,1}-\theta_{i, 1}\right)\right] \\
+ & 2 \times 2 J \sum_{i=2}^{6}\left[1-\cos \left(\theta_{i, 1}-\theta_{i, 0}\right)\right], \\
E_{I N T}= & s \times 2 J_{0}\left[1-\cos \left(\theta_{1,0}-\theta_{0,0}\right)\right]+(p-s) \\
& \times 2 J_{0}\left[1-\cos \left(\theta_{2,1}-\theta_{1,1}\right)\right] \\
& +2 \times 2 J_{0}\left[1-\cos \left(\theta_{1,1}-\theta_{1,0}\right)\right] .
\end{aligned}
$$

Furthermore, the weighting of the spins has to be taken into account for the writing of the anisotropy and Zeeman energy, $E_{K}$ and $E_{Z}$, respectively:

$$
\begin{gathered}
E_{K}=s \sum_{i=1}^{6} K \sin ^{2} \theta_{i, 0}+(p-s) \sum_{i=2}^{6} K \sin ^{2} \theta_{i, 1}, \\
E_{Z}=-s \sum_{i=-1,0} H \cos \theta_{i, 0}-(p-s) \sum_{i=-1}^{1} H \cos \theta_{i, 1} .
\end{gathered}
$$

Finally, the total magnetic energy $E_{T}$ is the sum over all different energy terms,

$$
E_{T}=E_{F}+E_{A F}+E_{I N T}+E_{K}+E_{Z} .
$$

It is worth noticing that the use of only two weighted spins on each atomic plane does not allow an accurate description of domain walls between both columns of spins or between spins within the AFM interfacial mesa and the FM layer. Indeed, for low-anisotropy value, the domain-wall width is expected to extend over several atomic planes. We made this choice in order to reduce the total number of spins in the system for computation time issues. However, as it will be shown, the anisotropy threshold above which the hysteresis field shift arises is such that the domain walls are very sharp under this circumstance, so that our assumption remains valid in this case.

Neglecting the coupling between the external field and the AFM spins is also an approximation that can stand for large anisotropy values only. Indeed, the applied field required to create a DW (a nucleation field) is of the order of $H_{n}=$ $\sqrt{J K} / M_{s}$. It represents the maximum of the energy that can be transmitted to the AFM layer via the interface. If the coupling between the external field $H$ and the AFM spins is taken into account, a field of the order of $H_{s}=\Delta \gamma / S$ is necessary to make the domain wall progress by an interatomic distance, where $\Delta \gamma$ and $S$ are the Peierls barrier and the AFM moment per atom, respectively. Therefore, as long as $H_{s} \gg H_{n}$, the coupling between the AFM spins and the external field can be neglected. The Peierls barrier strongly depends on the $K / J$ ratio; the sharper is the domain wall, the higher is the 
potential well. We estimated with the form of $\Delta \gamma$ that we previously derived (see Refs. [32,42]) that the approximation stands as long as $K / J>0.8$. Below this anisotropy threshold, the results are expected to be qualitatively different due to the torque of the external field on the AFM spins.

Two types of calculations are performed in this paper. By using the steepest-descent technique, we are able to evaluate both athermal $H_{E X}$ and $H_{C}$ with respect to $K$ and visualize the spin configuration within the AFM layer (presence and characteristics of the DWs). The general aim of the steepest-descent method is to follow a local minimum in the total magnetic energy from an initial configuration of the spins. Typically, once the initial configuration is chosen at the positive saturation, the applied field is decreased by small steps $\left(0.036 \times 2 J_{0} / M_{s}\right.$ in reduced units $)$ until the negative saturation is reached, and increased back to the positive one. For each field step, a new configuration of the spin angles is calculated: the angles $\theta_{i, j}$ are varied by $\delta \theta$ in the opposite direction of the local slope $\partial E_{T} / \partial \theta_{i, j}$. If this variation of the spin angles leads to a reduction of the total magnetic energy $\left(\delta E_{T}<0\right)$, then the new configuration is accepted, else $\delta \theta$ is divided by 10 and the procedure is run once again until the energy balance test is verified. With a $\delta \theta$ as small as $10^{-14}$, this method avoids the need to overcome the energy barriers. It is mostly dedicated to low-temperature study and is not adapted as soon as thermal activation has to be taken into account. That is why we also used the Monte Carlo method in order to calculate the thermal variation of $H_{E X}$ and $H_{C}$, and the possible training effect of both quantities, with respect to the temperature $T$. This probabilistic method is based on a random choice of the spin to be tilted within the structure, as well as a random choice of the tilt $\delta \theta$ itself. An energy balance test is also used with this method, but the probability of the acceptance of the event is given by the Boltzmann factor $\exp \frac{-\delta E_{T}}{T}$ if $\delta E_{T}>0$. This last procedure of random choices and balance test consists of a single Monte Carlo iteration; we usually performed $10^{5}$ iterations per spin and the magnetization is averaged over the last 1000 ones, for each field step $\left(0.2 \times 2 J_{0} / M_{s}\right.$ in reduced units). More details about both calculation methods are given in Ref. [42]; the only change in the present paper is the form of the total magnetic energy $E_{T}$ given by Eqs. (1)-(6). The system is set at the beginning of the calculations with the magnetization saturated along the positive $(O x)$ direction in a strong magnetic field. Since the FM exchange coupling considered here is larger than the coupling at the interface, or between AFM spins, the three initial configurations assumed in this paper only involve interfacial frustration or one atomic distance DW pinned in the first AFM planes. The energy cost $E_{C}$ of the initial configuration depends on $s$ and on the different exchange parameters. For $s<6$, a DW is pinned in the AFM mesa (configuration $c$ ). Above $s=6$, it is more favorable to create a magnetic frustration at the interface of the second column of spin (configuration $a$ ). Note that the energy of the $b$ configuration in Fig. 1 is very close to the $a$ one, so that the system will relax sometimes to this type of initial state for low- $K$ value, or under temperature effects. In order to correctly compare the critical fields obtained with the different initial configurations of the system, $H_{E X}$ and $H_{C}$ are normalized to $2 J_{0} / M_{s}$ (see Ref. [42] for details about the numerical determination of the critical fields). For large $K$,
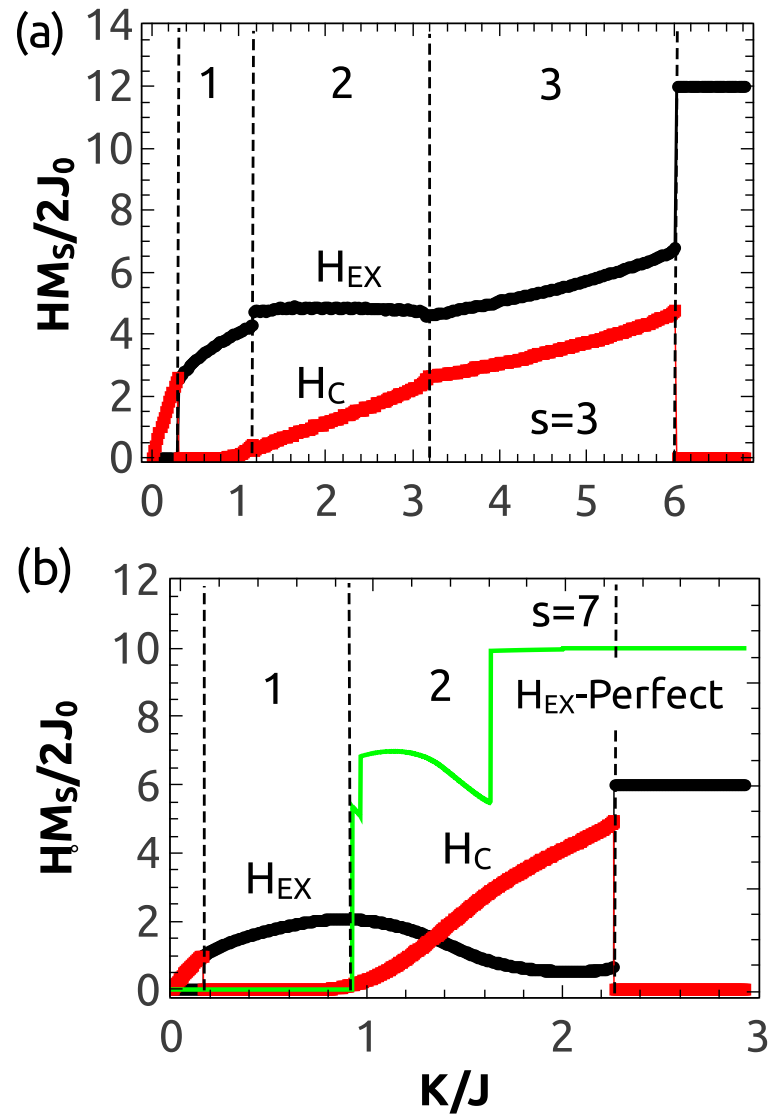

FIG. 2. Variation of the athermal normalized exchange bias $H_{E X}$ and coercivity $H_{C}$ with respect to $K / J$ for two initial configurations of the system, (a) $s=3$ and (b) $s=7$. For a large AFM mesa width in (b), the general trend of the $H_{E X}$ variation is very close to the perfect interface case plotted in the figure, with, however, another $K / J$ scale.

when the AFM layer is frozen, the dissipation is expected to vanish $\left(H_{C}=0\right)$ and the exchange bias to reach its maximal value, which is only related to the total exchange energy at the interface. Then the quantity $H_{E X} M_{s} / 2 J_{0}$ will refer for large $K$ to the number of FM/AFM coupled spins in the original setting direction, i.e., $2 s-p+2$ for configuration $a$, and 12 for configurations $b$ and $c$ as shown in Fig. 1. In the rest of the manuscript, the exchange bias will always be considered and plotted as a positive quantity, although the hysteresis loop shift along the field axis is negative.

\section{STEEPEST-DESCENT RESULTS}

In Fig. 2 are shown the variations of $H_{E X}$ and $H_{C}$ vs $K / J$ for two different system configurations, $s=3$ [Fig. 2(a)] and $s=7$ [Fig. 2(b)]. For $s=3$, in Fig. 2(a), five different regimes are clearly evidenced. For $K / J<0.3$, the entire AFM layer is reversed during both ascending and descending hysteresis branches, leading to no exchange bias and large coercivity. For $0.3<K / J<1.2$ [regime labeled 1 in Fig. 2(a)], a significant $H_{E X}$ arises with a small, but increasing $H_{C}$.

A characteristic hysteresis loop, as well as the FM/AFM spin configuration at the saturation fields corresponding to this first regime, are shown in Fig. 3(a) for $K / J=0.32$. When the applied field is swept down to the negative saturation, the 

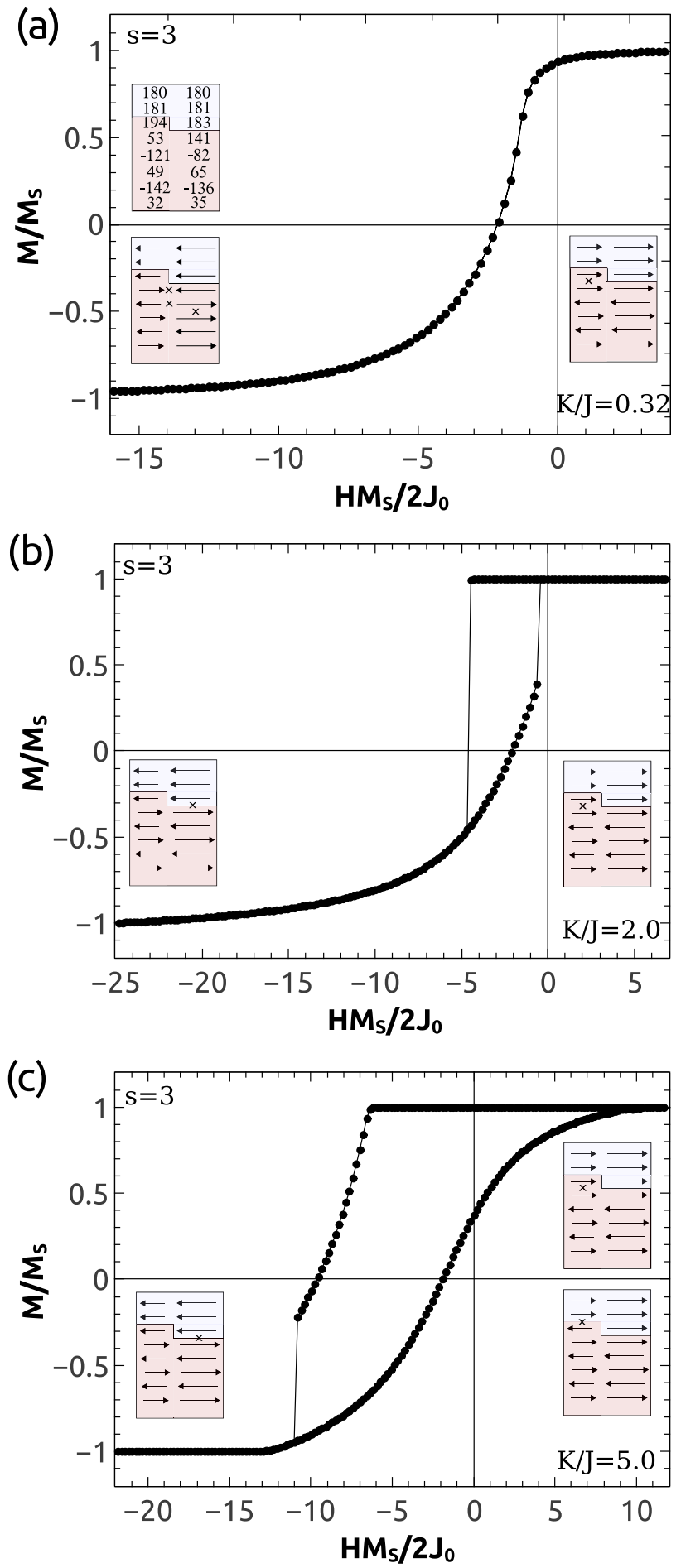

FIG. 3. Characteristic hysteresis loops calculated with the steepest-descent technique for $s=3$ and three different $K / J$ ratios. In the inset of the figures, on the right, the initial spin state in the setting conditions is shown, and on the left, the spin configuration reached at the negative saturation. In (c), the bottom low inset is the spin configuration at the end of the hysteresis loop. The crosses in the different insets emphasize the location of domain-wall centers or of magnetic interfacial frustrations. The top left inset in (a) presents the angles in degree reached by the spins in both FM and AFM layers at the negative saturation. In (b) and (c), the spins angles are aligned with the easy axis and then not specified in the figure.
DW pinned within the AFM interfacial mesa in the initial state is released (initial configuration $c$ in Fig. 1), and another DW is created in the second column in order to minimize the exchange energy at the interface. The opposite phenomenon arises when the applied field is increased back to the positive saturation. As depicted in the left inset of Fig. 3(a), the formation of a DW centered between $i=3$ and $i=4$ within the second column at the negative saturation also requires the formation of DWs between both columns. The spin angles within both columns at the negative saturation are given in the inset of Fig. 3(a) for clarity. For the low-anisotropy value shown in Fig. 3(a) $(K / J=0.32)$, the loop is almost reversible. However, coercivity arises as soon as $K / J>0.8$ as depicted in Fig. 2(a) with the same processes of DW annihilation/creation we just described above.

In the second regime, for $1.2<K / J<3.2$, the normalized $H_{E X}$ saturates around 5 [see Fig. 2(a)], while the coercive field increases significantly. In Fig. 3(b), it can be seen that, in this case, the AFM spins within the second column remain frozen in the initial configuration state, while the DW inside the AFM interfacial mesa is first depinned, and then created back, during the descending and ascending hysteresis branches, respectively. In that case, the spins are perfectly aligned with the AFM easy axis at the saturation.

For $3.2<K / J<6$, the same processes as in the second regime occur, when the applied field decreases to the negative saturation field. However, during the ascending branch of the hysteresis loop, the creation of a single atomic distance DW inside the AFM mesa costs more energy than breaking up the exchange at the interface. Therefore, the system remains frozen in the configuration reached at the negative saturation [see Fig. 3(c)]. This third regime is characterized by both increasing $H_{E X}$ and $H_{C}$ vs $K / J$, as depicted in Fig. 2(a). Above $K / J=6$, the entire AFM layer keeps its initial state structure because the energy required for the DW depinning is too large with respect to the total exchange energy at the interface. In such condition, the normalized $H_{E X}$ saturates to 12 and the coercive field falls down to zero as expected.

For the second configuration $(s=7)$, the variation of the critical fields is characterized by four regimes [see Fig. 2(b)]. Below $K / J=0.18$ and above $K / J=2.3$, the AFM spins are entirely reversed and remain frozen, respectively, for the same reason as for $s=3$. The normalized exchange bias in the frozen regime reaches the maximum value $2 s-p+2=6$, i.e., the total number of exchange coupled spins at the interface.

For $0.18<K / J<0.9$, in the regime labeled 1 in Fig. 2(b), a significant increase of $H_{E X}$ is evidenced. The corresponding AFM spins state is shown in the insets of Fig. 4(a). Although the system was originally set in configuration $a$, it relaxes to a configuration of type $b$ during the first calculation steps at the positive saturation. Actually, the choice of the initial state was made assuming only the exchange energy cost between the different possible configurations, when the spins are saturated along the easy-axis direction. In the present case, an energetic tradeoff is found by tilting the AFM spins in both columns, so that the configuration of type $b$ costs less than the $a$ one. As a consequence, there is also the formation of DWs between both columns on the first AFM planes at the positive saturation. When the applied field is decreased down to zero, the FM 

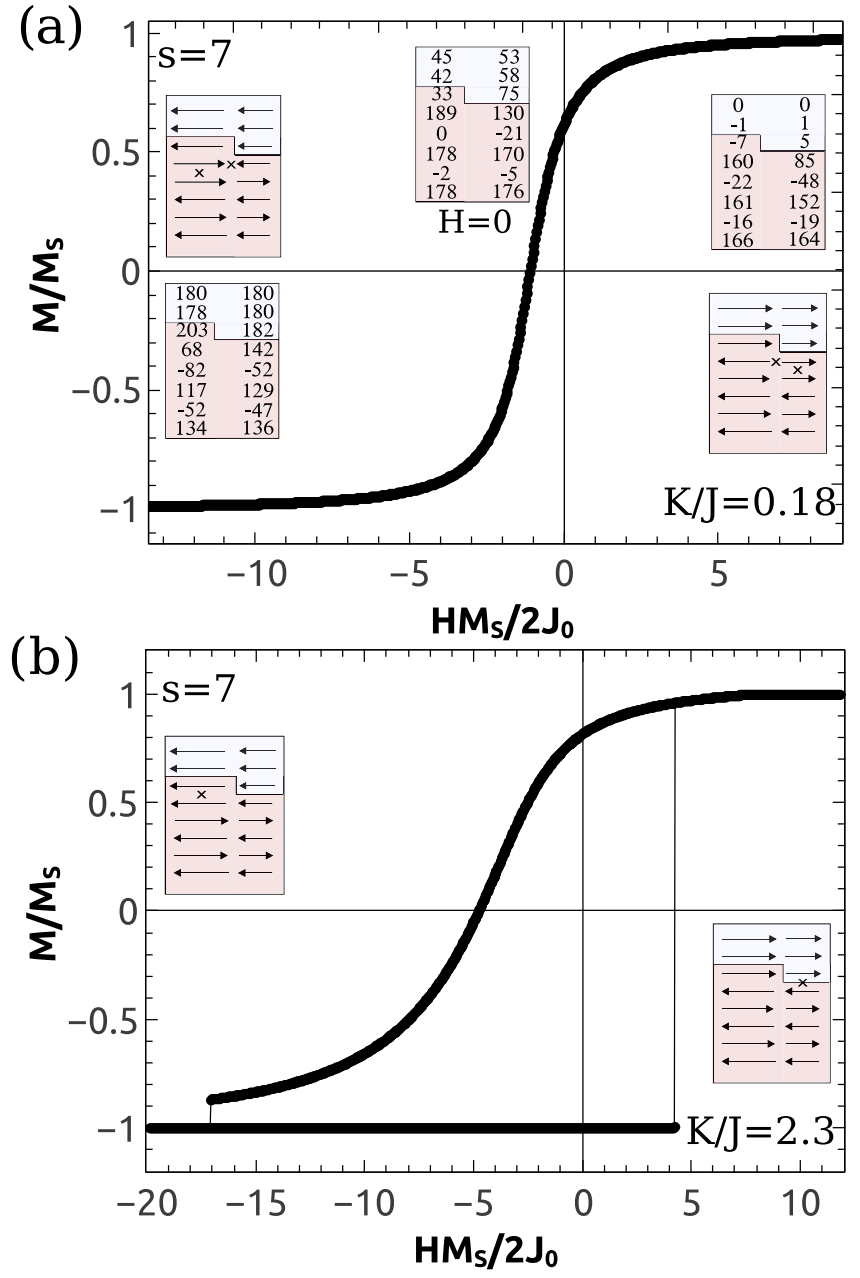

FIG. 4. Characteristic hysteresis loops calculated with the steepest-descent technique for $s=7$ and two different $K / J$ ratios in the two regimes of Fig. 2(b). The insets in (a) present the spin angles at the positive saturation (right), at the remanent state (middle), and at the negative saturation (left).

spins rotate in order to reduce the exchange energy cost at the interface, which leads to a reduction of the remanent magnetization. Finally, when the applied field is swept down to the negative saturation, the DW formed within the right column is depinned, while a 2 -atomic-distance DW is created in the left column. The opposite processes occur when the applied field is swept back to the positive saturation.

When $0.9<K / J<2.3$, the system cannot relax to the $b$-type configuration because the DW are very sharp (oneatomic-distance wide) for such high-anisotropy values [see Fig. 4(b)]. In this regime, labeled 2 in Fig. 2(b), a DW is created and then depinned inside the AFM mesa during the hysteresis loop, leading to a considerable increase of $H_{C}$ and a relative decrease of $H_{E X}$.

These athermal calculations show that DWs of two types can be formed in the AFM layer, parallel to the FM planes and in the AFM bulk, respectively, in order to reduce the exchange energy cost at the interface. The dissipation is always due to the depinning of sharp DWs from Peierls potential wells, as we already showed in our previous studies.
However, the depinning processes can happen in the present case on both hysteresis branches, depending on the initial configuration. This is only possible for intermediate $K / J$ values though, where the formation of DWs is energetically favorable. Moreover, the presence of the atomic mesa at the interface leads to the formation of DWs between both columns of spins, which did not occur in the case of perfect interface (although more than two columns of spins are necessary to accurately describe such DWs, as we previously mentioned). This is consistent with Malozemoff's random field approach, who postulated the creation of reversed bubbles in the AFM bulk, implying the existence of these two types of magnetic walls [11] in the presence of atomic roughness. Finally, the presence of the interfacial roughness also leads to a reduction of the anisotropy threshold beyond which DWs can be stabilized within the AFM layer. As can be seen in Fig. 2(b), where the variation of $H_{E X}$ vs $K / J$ is plotted in the case of flat interface (three FM planes/five full AFM planes), the DW regime arises for $K / J>0.9$. This is larger than for $s=3$ $(K / J>0.3)$ [Fig. 2(a)] and $s=7(K / J>0.18)$ [Fig. 2(b)] and for any rough configuration we have investigated. It can be explained by the lateral exchange interactions between the two columns that help to stabilize short DWs within the AFM layer.

\section{MONTE CARLO RESULTS}

\section{A. Thermal variation}

In order to study the thermal evolution of both $H_{E X}$ and $H_{C}$, we performed Monte Carlo (MC) simulations on the same system, for $s$ varying between 1 and 9 . For each temperature, about 100 hysteresis loops are calculated and the characteristic fields averaged on all of them. The system is set in the initial state corresponding to configurations $a$ and $c$ as previously shown at the beginning of the simulations. We set the ratio $K / J=5.6(J=-0.5)$ and kept it constant for all $T$ and for all investigated values of $s$. In Fig. 5(a), the normalized $H_{E X}$ and $H_{C}$ vs $T / J$ are shown for $s=3$. A decrease of $H_{E X}$ with $T$, showing three different regimes below the blocking temperature $\left(T_{B}=2.6 \mathrm{~J}\right)$, is evidenced.

The corresponding averaged hysteresis loops in the regimes labeled from 1 to 3 in Fig. 5(a), as well as at high temperature, are shown in Fig. 6. It is clear in Fig. 6(a) that the loops are very similar to what was calculated using the steepest-descent technique for $0.3<K / J<6$ [compare Fig. 3 with Fig. 6(a)], leading to similar values of $H_{E X}$ and $H_{C}$. As a matter of fact, the same processes as previously explained occur within the AFM layer in the three regimes of Figs. 5(a) and 2(a). A first effect of the temperature is then to decrease the effective thermal AFM anisotropy with respect to its nominal value.

The transition between the first regime of Fig. 5(a) and the high temperatures is characterized by a decrease of $H_{E X}$ up to $T_{B}$, where the coercivity peaks. In this range of temperature, the calculated hysteresis loops are a statistical combination of exchange biased and symmetrical loops [see the curve for $T / J=2.44$ in Fig. 6(b)]. As we already demonstrated in our previous study [42], the thermal activation induces a random walk of the sharp DWs within the AFM layer. This leads to the entire reversal of the AFM spins when the DW is expelled out from the layer. 


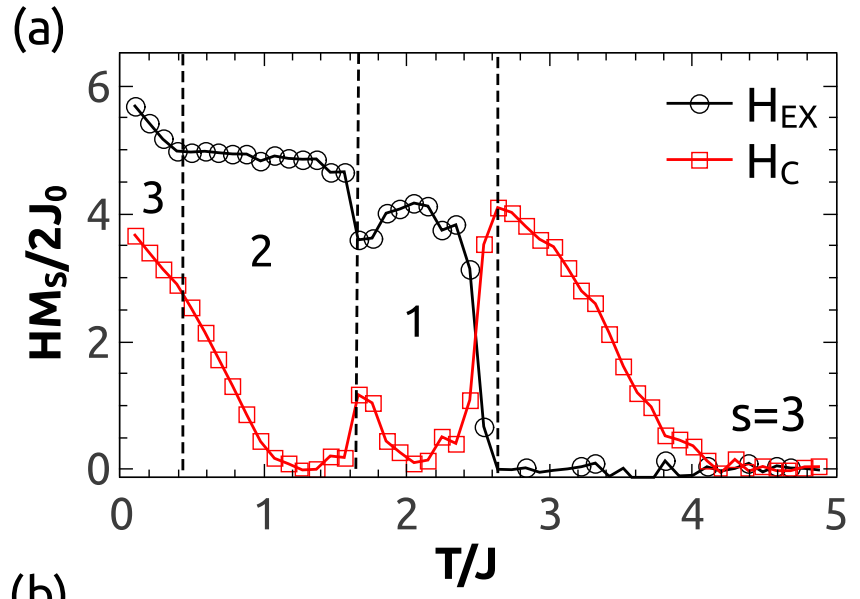

(b)

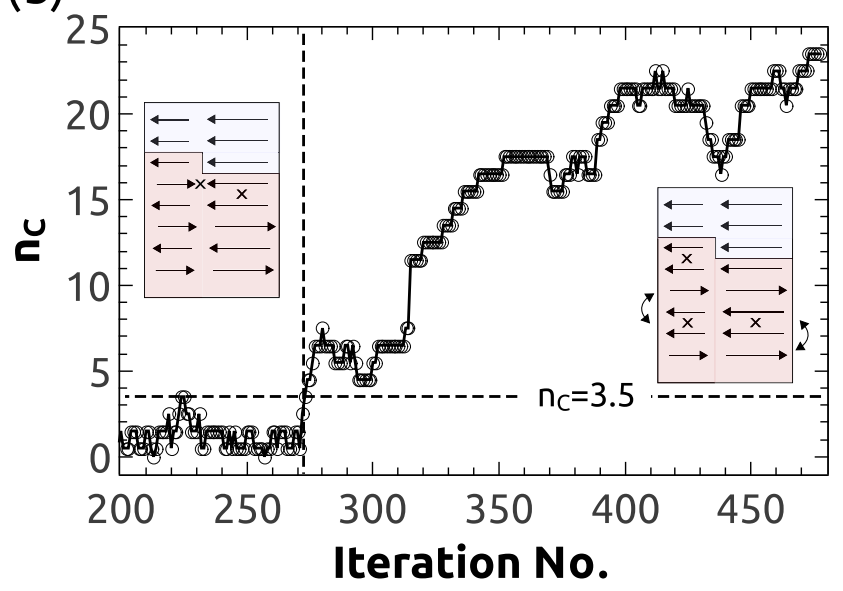

FIG. 5. (a) Thermal variation of $H_{E X}$ and $H_{C}$ for $s=3$ calculated with the Monte Carlo method. The ratio $K / J$ is kept constant during the simulations to 5.6. The three regimes depicted in the figure correspond to the regimes evidenced in Fig. 2(a). (b) Position of the DW center $n_{C}$ with respect to the iteration time at $T / J=3$ and $H M_{s} / 2 J_{0}=-15$. The simulations are performed on an AFM layer 25-atomic-planes thick in order to emphasize the random walk of the DW on a larger scale. The inset in the left side of the figure shows the spin configuration just after the magnetization reversal. The DW center is located in this case between $i=2$ and $i=3$ within the right column of spins $\left(n_{C}=2.5\right)$. On the right side of the figure, the inset exhibits the presence of the same DW, but extending over the two columns. Its center has jumped several maxima of the Peierls potential, driven by thermal activation. This configuration implies the creation of another DW within the AFM mesa.

In Fig. 5(b), the left inset shows the AFM spin configuration reached at the negative saturation for $T / J=3$ and $s=3$. A sharp DW is pinned in the right column of spins at the position $n_{c}=2.5$ (between $i=2$ and $i=3$ ). During the calculations, the DW center $n_{c}$ jumps from one equilibrium position to another by thermal activation, as depicted in Fig. 5(b). We present in the figure the results for a 25-atomicplane-thick AFM layer, just to emphasize the random walk over a larger scale. When the DW reaches the position $n_{c}=4.5$ [see the right inset of Fig. 5(b)], there is creation of another DW inside the AFM mesa, in order to minimize the lateral exchange interactions between the two columns of spins. This results in a DW extending over both columns, which progresses
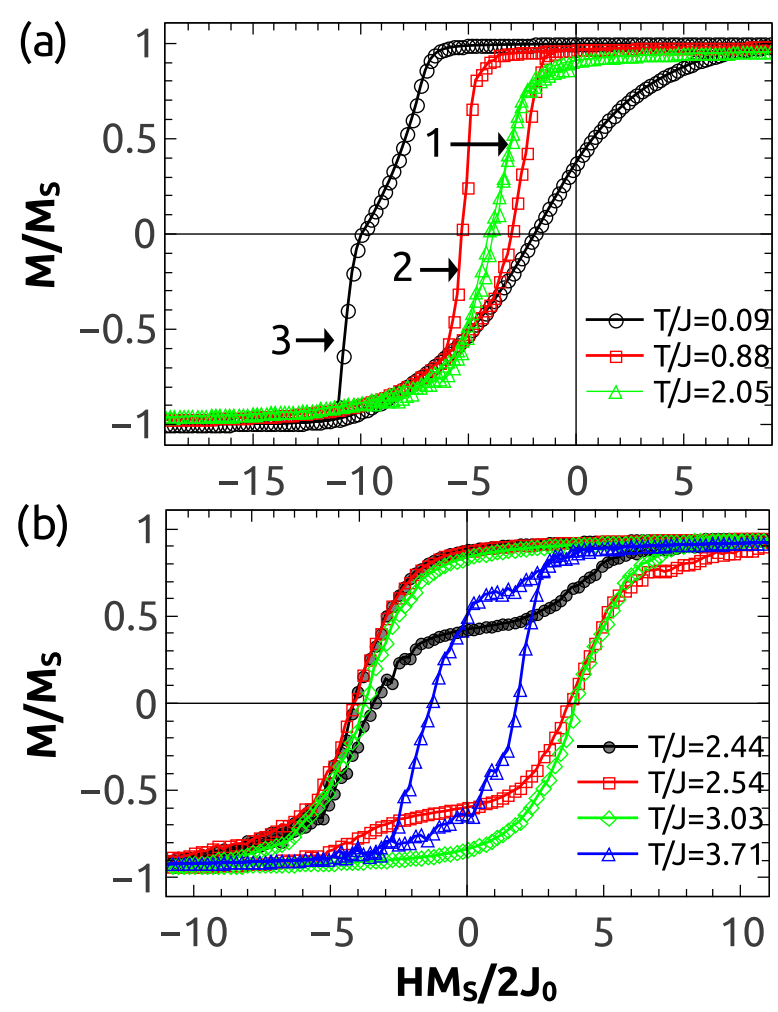

FIG. 6. Hysteresis loops calculated using the Monte Carlo method for different $T / J$ ratio. In (a), the loops are calculated on the three regimes of Fig. 5(a).

throughout the AFM layer as depicted in the figure, while the second one stays pinned inside the AFM mesa. When $T$ is increased, the probability for the DW to be expelled out from the AFM layer during the descending hysteresis branch increases, which leads to a reduction of $H_{E X}$ and a significant increase of $H_{C}$, as shown in Fig. 5(a) for $T / J>2.2$. Above $T_{B}$, the systematic reversal of the AFM spins only induces coercivity, which tends to disappear at high temperature when the effective thermal AFM anisotropy vanishes. The result presented in Fig. 5(b) is extracted from a single hysteresis loop and is not an averaged measurement. If the simulation is run once again with the same magnetic parameters, initial configuration, and temperature, the path taken by the DW through the AFM layer will be different because of its random walk. The final result, i.e., the domain wall extending onto both columns of spins still present within the AFM layer or expelled from it, will also vary, depending on the temperature. That is why it is necessary to carry out a large amount of simulations to reach a stable averaged value of the critical fields.

The same kind of processes, i.e., reduction of the effective thermal AFM anisotropy and random walks of magnetic DWs, arises for all of the investigated configurations. Using the characteristic fields vs $T$ for $s=1$ to 9 obtained with the MC method, we are able to calculate the average behavior for a rough interface, as shown in Fig. 7. When the AFM atoms are the minority species at the interface on the plane $i=1$ [fields averaged for $1 \leqslant s \leqslant 5$ in Fig. 7(a)], the thermal variations of both $H_{E X}$ and $H_{C}$ reproduce fairly well the experimental measurements $[44,45]$. 

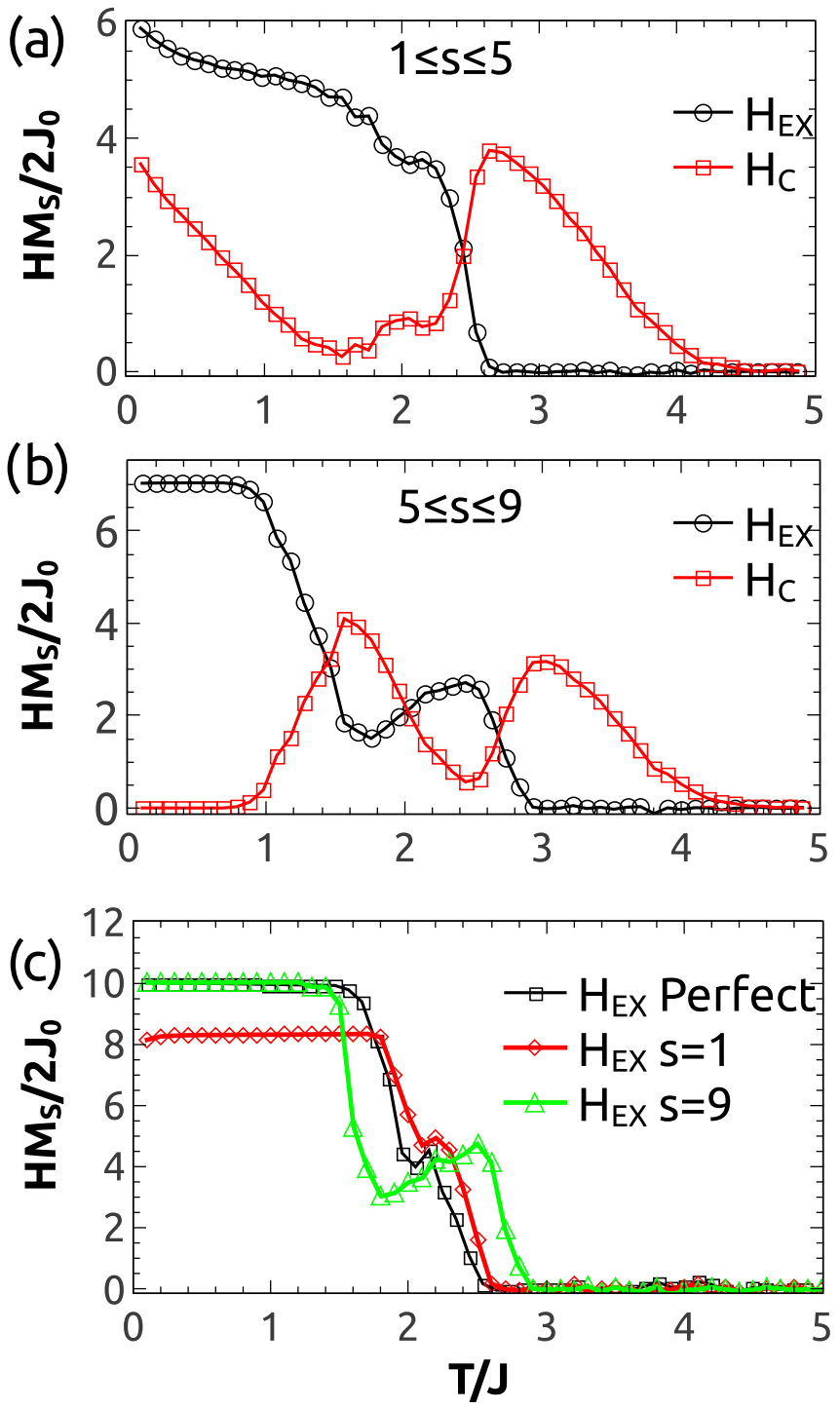

FIG. 7. Averaged normalized $H_{E X}$ and $H_{C}$ calculated using the Monte Carlo method. (a) The fields are averaged for $1<s<5$, describing the case where the AFM atoms are the minority species $(s<5)$ at the FM/AFM interface on the plane $i=1$. (b) The fields are averaged for $5<s<9$. (c) Results for $s=1$ and $s=9$ compared to the perfect interface case.

When $5 \leqslant s \leqslant 9$ [Fig. 7(b)], the average thermal behavior of both fields is qualitatively close to the perfect interface case, with, however, another temperature scale (see Fig. 7(c) as well as Fig. 6 in Ref. [42]). We also plotted in Fig. 7(c) the two extreme cases corresponding to $s=1$ and $s=9$. For $s=9$, the initial AFM configuration is the one depicted in Fig. 1(a). At low temperature, when the AFM spins remain frozen during the hysteresis loop, the exchange bias corresponding to this $a$ state is of the order of $2 s-p+2=10$ in reduced units, corresponding to the regime $T / J<1.8$ in the figure. For $s=1, H_{E X}$ is expected to reach 12 in reduced units when no thermal activation arises. As it can be seen in the figure, the exchange bias for $s=1$ is about 8 at very low $T$. It is because the anisotropy set in the Monte Carlo simulations $(K / J=5.6)$ is not large enough to freeze the AFM spins during the hysteresis loop, i.e., there is still depinning/formation of DW in the AFM layer for such a value of the anisotropy and $s=1$. That is why when the exchange bias is averaged over all the mesa sizes investigated, its amplitude is decreased with respect to the perfect interface case, as already evidenced by other authors $[11,46]$. Furthermore the use of normalized units, here $H M_{s} / 2 J_{0}$, allows the extrapolation of the critical fields for other FM thickness: both are inversely proportional to the total magnetic moment of the FM layer. Concerning the evolution of our results with the AFM thickness, we can guess, as shown in our previous study [42], that the thermal evolution will be quite similar, although another temperature scale has to be considered. This argument is supported by the fact that the case $s=9$, where the AFM layer is almost one atomic plane thicker than the case $s=1$, presents a similar thermal evolution, with an increased blocking temperature [see Fig. 7(c)]. Note that in Fig. 7(c), the perfect interface case is presented for five AFM planes, and exhibits also lower $T_{B}$ than for $s=9$. We did not investigate other periodicity than $p=10$, but we think that the processes evidenced here will be qualitatively comparable, besides the fact that the initial configuration of the AFM spins depends on the ratio between $p$ and $s$ and that configuration $b$ will be preferred to configuration $a$ for larger periodicity and $s>p / 2$. For larger periodicity also, the contribution of the exchange interaction of both sides of the AFM mesa with the FM spins $\left(2 \times 2 J_{0}\right)$ will decrease with respect to the total interfacial exchange coupling in the $(O y)$ direction $\left[p \times 2 J_{0}\right.$ or $(2 s-p) \times 2 J_{0}$, depending on the initial configuration]. Then when $p>>2$ and $s<$ $p / 2$ (configuration $c), E_{I N T} \simeq(p+2) \times 2 J_{0} \simeq p \times 2 J_{0}$. For larger mesa size, $s>p / 2$, configurations $a$ or $b$ are preferred and $E_{I N T}$ still tends to $p \times 2 J_{0}$, i.e., to the perfect interface case.

Finally, we performed a careful error analysis of the data for all measurements presented in this paper. A first source of error affecting the value of the characteristic fields is due to the numerical integration method used to extract $H_{E X}$ and $H_{C}$ from the averaged hysteresis loops. This uncertainty mainly comes from the finite applied field step of the Monte Carlo simulations which is of about $0.2 \times 2 J_{0} / M_{s}$ expressed in the reduced unit of the figures. Moreover, the fact that the depinning of the domain walls from the Peierls potential wells and their random walk through the AFM layer is thermally activated induces a natural dispersion of the critical fields associated with the magnetization reversal. We evaluated this dispersion to $0.09 \times 2 J_{0} / M_{s}$ on a series of individual hysteresis loops. That is why, for all thermal variations presented in this article, the error bars are approximately of the size of the symbols used in the figures $\left(\simeq 0.2 \times 2 J_{0} / M_{s}\right)$.

\section{B. Training effect}

We studied the influence of performing $n$ successive hysteresis loops on the AFM spin configuration and on the exchange bias and coercivity for different temperatures. We focus on the case $s=3$ in the same conditions as previously done.

In Fig. 8(a), the normalized $H_{E X}$ thermal variation is shown for five successive hysteresis loops. At low $T$, for $T / J<0.5, H_{E X}$ drops by a factor 3 between the first and 
(a)
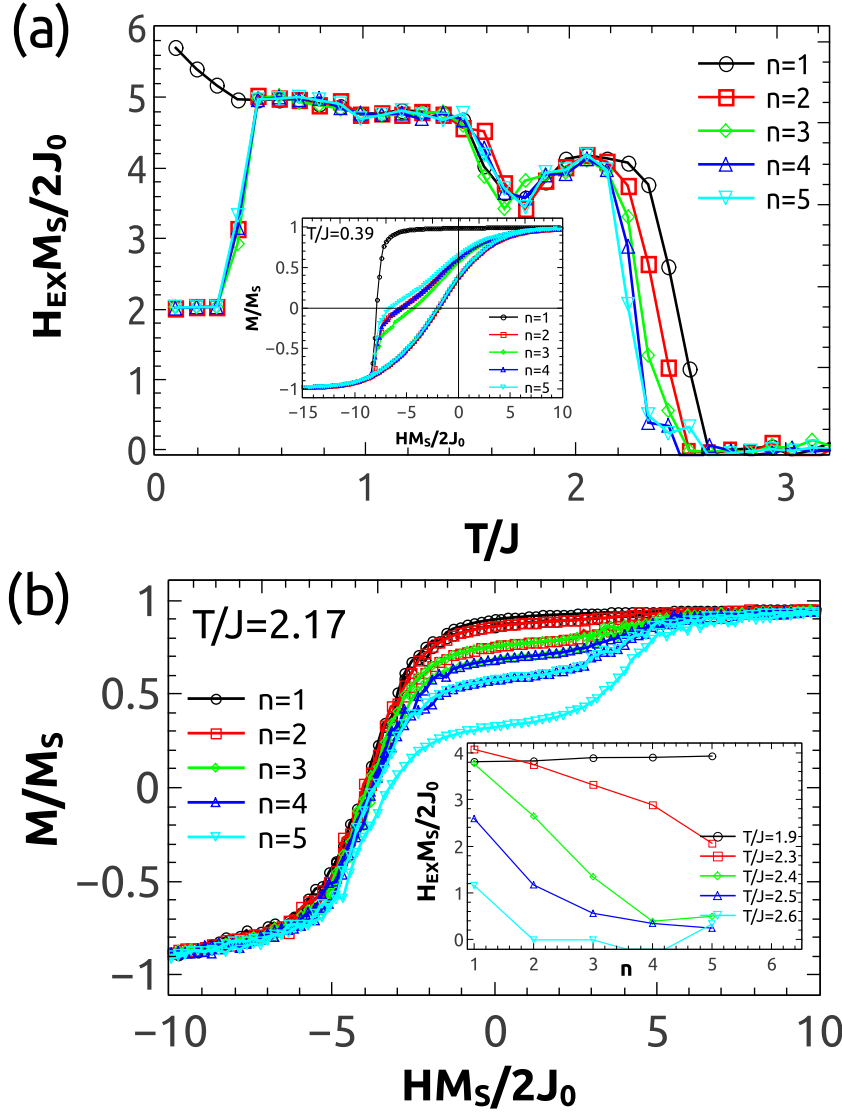

FIG. 8. (a) Thermal variation of the normalized $H_{E X}$ vs $T / J$ for the five successive hysteresis loops. In the inset, the characteristic loops obtained at low temperature for $T / J=0.9$ are shown. (b) Successive hysteresis loops calculated at $T / J=2.17$. In the inset, the variation of the normalized $H_{E X}$ vs the loop number $n$ is shown for different $T / J$ ratios.

the second loop and then remains constant for $n \geqslant 2$. For intermediate temperature, no training effect is evidenced, while for $2.2<T / J<2.6$, a constant and relative decrease of $H_{E X}$ vs $n$ is obtained. The successive loops at low $T$ are depicted in the inset of Fig. 8(a). At low temperature, the effective thermal AFM anisotropy is comparable to the nominal one $(K / J=5.6)$. It corresponds to the third regime of Fig. 2(a): the sharp DW stabilized in the Peierls potential well within the AFM mesa (configuration $c$ ) is depinned during the descending branch of the hysteresis loop. During the ascending branch though, the energetic cost of formation of such very thin wall is larger than the total exchange energy at the interface. As a result, the system remains frozen in the state reached at the negative saturation. Consequently, the initial configuration of the second loop is not the $c$ one, but exhibits a frustration between the AFM mesa and the FM layer, as depicted in the inset of Fig. 3(c). It leads to a normalized $H_{E X}$ equal to $p-2 s-2=2$ for $n \geqslant 2$. This calculated behavior at low temperature as well as the hysteresis loops shape shown in Fig. 8(a) (inset) are very close to the experimental results [47].

Just below the blocking temperature, the training of the exchange bias is also significant. In Fig. 8(b) are shown the five successive averaged hysteresis loops for $T / J=2.17$ (averaged over all of the 100 calculated loops per temperature). (a)
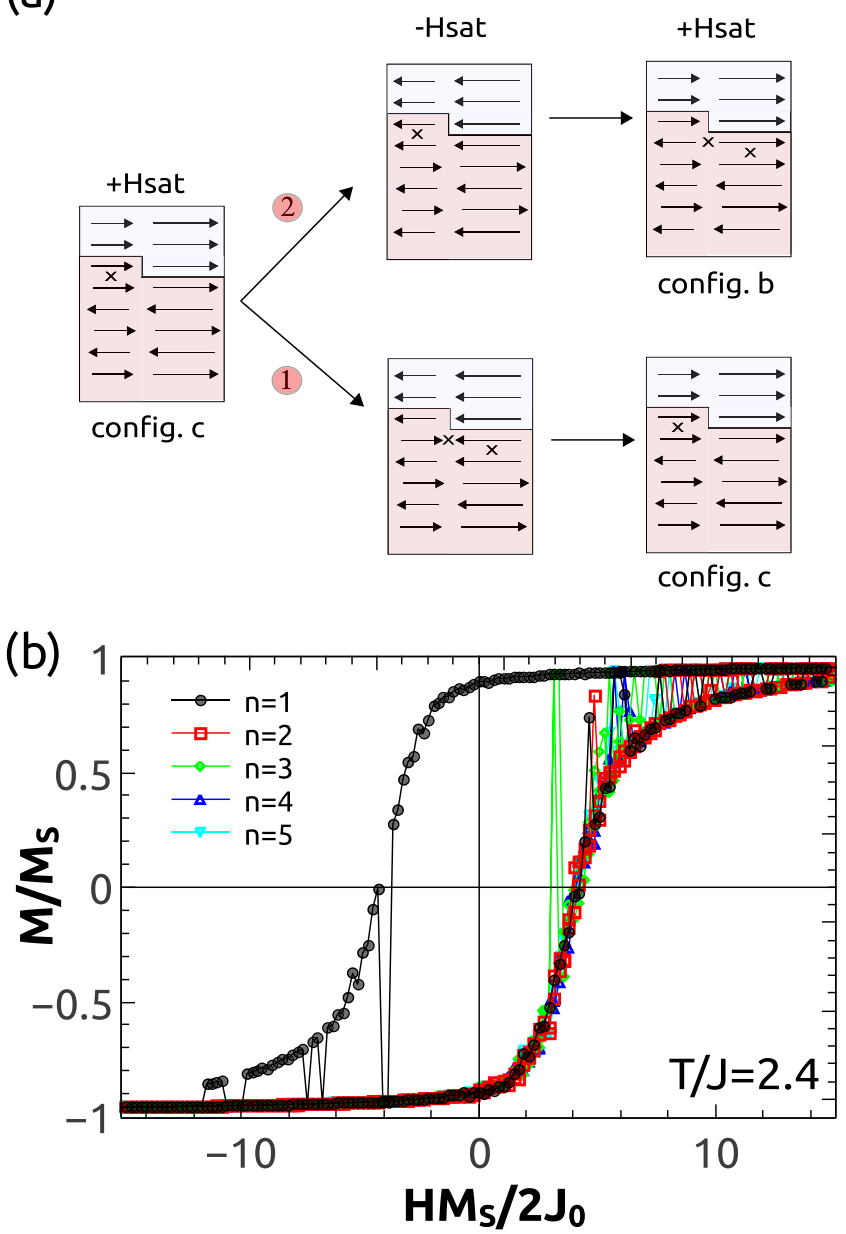

FIG. 9. (a) Sketch of two different spin configurations obtained after the first hysteresis loop, when the system is set in the $c$ configuration at the beginning of the calculations. If the DW is expelled out from the AFM layer at the negative saturation (case 2), a $b$-type configuration is found at the beginning of the second loop. (b) Successive hysteresis loops calculated using the Monte Carlo method at $T / J=2.4$. The loops presented in the figure are a single result of the 100 loops calculated per temperature. The first cycle is completely open and centered and corresponds to the path labeled 2 of Fig. 9(a). At the beginning of the following loops, the system is in a $b$-type configuration, which leads to a positive exchange bias.

The first averaged loop is reversible and exhibits a large exchange bias of the order of 4 in normalized units. When $n>1$, the hysteresis loop shift tends to decrease and the coercivity to increase with $n$. In this temperature range, we demonstrated in the previous section that the DWs undergo a random walk through the AFM layer [see Fig. 5(b)]. The AFM spin configuration at the negative saturation depends on the probability for the wall to be expelled out from the AFM layer. If the DW is still located within the AFM layer when the external field is swept back to the positive saturation, then the initial configuration is unchanged at the beginning of the next hysteresis loop. This case is described by the path labeled 1 in the sketch of Fig. 9(a). It leads to a classical negative field shift of the hysteresis loop. On the other hand, if the DW extending over the two columns is expelled out from the AFM layer, an 
atomic distance DW is pinned within the AFM mesa at the negative saturation as depicted in Fig. 9(a) (path labeled 2). During the ascending branch of the hysteresis loop, the torque exerted by the magnetization unwinds the DW from the AFM mesa, while a sharp DW is created within the right column. This leads to a $b$ configuration type at the beginning of the second loop, and to an open and centered hysteresis loop.

In Fig. 9(b), five successive hysteresis loops are shown (single loops out of the 100 calculated per temperature) obtained when the DW extending over both columns is expelled out from the AFM layer during the first loop for $T / J=2.4$. An open and centered first hysteresis loop is followed by four others, which are shifted in the positive field direction, giving rise to an exchange bias opposite to the expected one, when the system is set in the initial $c$ configuration. This can be easily explained by a qualitative argument: for the $c(b)$ configuration in this range of anisotropy, during the descending hysteresis branch, a DW of width $s(p-s)$ must be depinned, and another one of width $p-s(s)$ formed at the FM/AFM interface. The reciprocal processes arise during the ascending hysteresis branch for both configurations. That is why the hysteresis field shift is opposite when the system is initially in configuration $b$ with respect to configuration $c$ for a given $s$. It is important to notice also that the final configuration ( $b$ or $c$ ) has to be the same as the initial one for observing positive or negative exchange bias. Indeed, starting with a configuration of type $b$ can lead to a configuration of type $c$ at the end of the hysteresis loop because of similar processes of DW training as depicted in Fig. 5(b): in this case, just like in the path labeled 2 of Fig. 9(a), the loops are open and centered. For the mesa size investigated here $(s=3)$, the system is set in the most energetically favorable $c$ configuration in all of the 100 samples that will be cycled by the applied field (100 calculations are achieved for each temperature value and each loop number). After the first loop, it is found that a proportion of the 100 considered samples is back into a $c$ state, while others have flipped into a $b$ one. This leads to a negative exchange bias contribution from the samples which have not flipped their initial state and to coercivity only from the others. After the second loop, the situation is a bit different. The samples originally in the $b$-type configuration can lead to a positive exchange bias or coercivity depending on their final configuration. Then during the successive hysteresis loops, the proportion of the initial $c$ configuration decreases in favor of the $b$ one in the 100 considered samples. It leads statistically to a reduction of $H_{E X}$ vs $n$ as expected, which could be in fact modeled by a binomial law. In the inset of Fig. 8(b), the variation of the normalized $H_{E X}$ vs $n$ is shown for increasing temperatures. The training effect is enhanced at higher temperature, which is due to the faster loss of the initial $c$ configuration with respect to $n$ (the temperature activates the random walk of DW and the occurrence of the $b$ configuration). Note that the jumps in the values of the magnetization occurring at different applied fields (for instance, at $H M s / 2 J_{0}=-4$ ) in Fig. 9(b) are probably due to rapid changes in the AFM configuration during the Monte Carlo iterations performed per field step, activated by the high temperature. While it is not possible to extract all the spin angles for all the simulated loops, one can guess that during the field step at $H M s / 2 J_{0}=-4$, the AFM order changes from a $c$ configuration to a $b$ one. This leads to a sudden alignment of the FM spins with the AFM easy axis, as loops number 2 to 5 in the same figure. During the next field step, the previous configuration is retrieved and the magnetization rotates back to its expected angle for this field value. Such rare events induce noise in the loop measurements that can be smeared out by increasing the sampling statistics.

\section{CONCLUSION}

The effect of atomic roughness at the FM/AFM interface was studied in the context of short DW pinned within Peierls potential wells. Different initial configurations of the AFM spins were investigated with respect to the interfacial mesa width. Assuming exchange energy between FM spins larger than between AFM ones, the roughness favors the stabilization of a sharp DW in the first AFM planes or magnetic frustration at the interface.

When the AFM mesa width is large with respect to the half of the system period $(s>5)$, the average behavior of the system is very similar to the flat interface case, though the temperature scale (or the $K / J$ ratio scale in the athermal calculations) is different. This difference arises from the lateral exchange between the two columns of spins at high temperature (or low $K / J$ ratio), as well as from the magnetic frustration at the interface for low temperatures (or high $K / J$ ratio).

For reduced AFM mesa width, the AFM atoms being the minority species at the interface $(1 \leqslant s \leqslant 5)$, a sharp DW is pinned within the AFM mesa in the initial state. This can lead to irreversible processes at low temperature (or high $K / J$ ratio), with the DW being unwound during the descending hysteresis branch but not formed back on the ascending branch. Both large exchange bias and coercivity arise from this irreversible process and can explain the large increase of both critical fields observed experimentally at low temperature [44]. When the system is at the negative saturation, for such a mesa width $(1 \leqslant s \leqslant 5)$ and for $T / J>1$, the AFM reversal can be achieved by a random walk of a sharp DW extending over the two spin columns. The probability for the DW to be expelled out from the AFM layer increases with $T$, which explains the reduction of the hysteresis loop shift just below $T_{B}$. In this range of temperature, one can expect that DWs would extend over several atomic planes. Our two-weighted-columns model would then fail to accurately describe DWs within the AFM bulk as explained in Sec. II. However, some authors [48] have shown that when $K / J>4 / 3$, DWs are of an Ising type, i.e., they extend over a single interatomic distance, for any temperature. As a matter of fact, we did not observe larger DWs in our Monte Carlo simulations, neither in the perfect interface case nor in the context of the present paper. Nonetheless, further studies are needed with a larger number of spins on each atomic plane in order to conclude more precisely about the effect of $p$ and $s$ in the whole range of temperature and anisotropy. Our study focused on roughness-periodicity and mesa size - at the atomic scale only and in the high-anisotropy regime of AFM layers.

The random-walk processes also induce a change in the initial spin configuration, when the system is back to the positive saturation, if the DW has been expelled out from the AFM layer. When several successive hysteresis loops are performed, training effects appear both at low $T$ and around 
the blocking temperature, as observed experimentally [45]. The low- $T$ contribution arises from irreversible processes within the AFM mesa. Our observation supports some other studies, which emphasized that the athermal training derives solely from interfacial spin configuration [29]. The calculated hysteresis loops reproduce also fairly well the characteristic changes in the loop shape measured experimentally. Finally, the thermally activated training effect, just below $T_{B}$, is found to be connected to the change in the bulk AFM spin configuration versus loop number.
The presence of atomic roughness at the FM/AFM interface allows the reproduction of two important experimental features of the exchange bias phenomenon, i.e., the thermal variation of the field shift and the coercivity, as well as the training effect. The interfacial roughness also reduces the exchange bias amplitude with respect to the perfect interface case by almost a factor of 2. As we already showed in our previous studies, by using realistic magnetic parameters for the anisotropy and the exchange energy, a relatively good agreement can be found between the calculated and measured $H_{E X}$ and $H_{C}$.
[1] B. Dieny, V. S. Speriosu, S. Metin, S. S. P. Parkin, B. A. Gurney, P. Baumgart, and D. R. Wilhoit, J. Appl. Phys. 69, 4774 (1991).

[2] W. H. Meiklejohn and C. P. Bean, Phys. Rev. 102, 1413 (1956).

[3] R. L. Stamps, J. Phys. D: Appl. Phys. 33, R247 (2000).

[4] J. Nogués and I. K. Schuller, J. Magn. Magn. Mater. 192, 203 (1999).

[5] A. E. Berkowitz and K. Takano, J. Magn. Magn. Mater. 200, 552 (1999).

[6] M. Kiwi, J. Magn. Magn. Mater. 234, 584 (2001).

[7] J. Nogués, J. Sort, V. Langlais, V. Skumryev, S. Suriñach, J. Muñoz, and M. Baró, Phys. Rep. 422, 65 (2005).

[8] V. Baltz, G. Gaudin, P. Somani, and B. Dieny, Appl. Phys. Lett. 96, 262505 (2010).

[9] G. Vinai, J. Moritz, G. Gaudin, J. Vogel, M. Bonfim, F. Lanon, I. L. Prejbeanu, K. Mackay, and B. Dieny, J. Phys. D: Appl. Phys. 46, 345308 (2013).

[10] W. H. Meiklejohn and C. P. Bean, Phys. Rev. 105, 904 (1957).

[11] A. P. Malozemoff, Phys. Rev. B 35, 3679 (1987).

[12] U. Nowak, K. D. Usadel, J. Keller, P. Miltényi, B. Beschoten, and G. Güntherodt, Phys. Rev. B 66, 014430 (2002).

[13] E. Fulcomer and S. H. Charap, J. Appl. Phys. 43, 4190 (1972).

[14] K. O'Grady, L. E. Fernandez-Outon, and G. Vallejo-Fernandez, J. Magn. Magn. Mater. 322, 883 (2010).

[15] G. Vallejo-Fernandez, L. E. Fernandez-Outon, and K. O'Grady, J. Phys. D: Appl. Phys. 41, 112001 (2008).

[16] M. D. Stiles and R. D. McMichael, Phys. Rev. B 63, 064405 (2001).

[17] M. D. Stiles and R. D. McMichael, Phys. Rev. B 59, 3722 (1999).

[18] T. C. Schulthess and W. H. Butler, Phys. Rev. Lett. 81, 4516 (1998).

[19] C. Leighton, J. Nogués, B. J. Jønsson-Åkerman, and I. K. Schuller, Phys. Rev. Lett. 84, 3466 (2000).

[20] V. Baltz, B. Rodmacq, A. Zarefy, L. Lechevallier, and B. Dieny, Phys. Rev. B 81, 052404 (2010).

[21] D. Paccard, C. Schlenker, O. Massenet, R. Montmory, and A. Yelon, Phys. Status Solidi 16, 301 (1966).

[22] J. Keller, P. Miltényi, B. Beschoten, G. Güntherodt, U. Nowak, and K. D. Usadel, Phys. Rev. B 66, 014431 (2002).

[23] C. Schlenker and D. Paccard, J. Phys. (Paris) 28, 611 (1967).

[24] M. Fecioru-Morariu, J. Wrona, C. Papusoi, and G. Güntherodt, Phys. Rev. B 77, 054441 (2008).
[25] A. Hoffmann, Phys. Rev. Lett. 93, 097203 (2004).

[26] M. K. Chan, J. S. Parker, P. A. Crowell, and C. Leighton, Phys. Rev. B 77, 014420 (2008).

[27] H. Xi, R. M. White, S. Mao, Z. Gao, Z. Yang, and E. Murdock, Phys. Rev. B 64, 184416 (2001).

[28] K. Zhang, T. Zhao, and H. Fujiwara, J. Appl. Phys. 89, 6910 (2001).

[29] B. Kaeswurm and K. O’Grady, Appl. Phys. Lett. 99, 222508 (2011).

[30] L. Néel, Ann. Phys. (Paris) 2, 61 (1967).

[31] D. Mauri, H. C. Siegmann, P. S. Bagus, and E. Kay, J. Appl. Phys. 62, 3047 (1987).

[32] J. Moritz, P. Bacher, and B. Dieny, Phys. Rev. Lett. 112, 087201 (2014).

[33] J. O. Artman, J. C. Murphy, and S. Foner, Phys. Rev. 138, A912 (1965).

[34] N. P. Aley, M. Bowes, R. Kröger, and K. O’Grady, J. Appl. Phys. 107, 09D722 (2010).

[35] N. P. Aley, R. Kroeger, B. Lafferty, J. Agnew, Y. Lu, and K. O’Grady, IEEE Trans. Magn. 45, 3869 (2009).

[36] H. R. Hilzinger and H. Kronmüller, Phys. Status Solidi B 54, 593 (1972).

[37] T. Egami and C. D. Graham, J. Appl. Phys. 42, 1299 (1971).

[38] J. J. van den Broek and H. Zijlstra, IEEE Trans. Magn. 7, 226 (1971).

[39] B. Barbara, J. Magn. Magn. Mater. 129, 79 (1994).

[40] J. I. Arnaudas, A. Del Moral, and J. S. Abell, J. Magn. Magn. Mater. 61, 370 (1986).

[41] H. Kronmüller, J. Magn. Magn. Mater. 7, 341 (1978).

[42] J. Moritz, P. Bacher, and B. Dieny, Phys. Rev. B 90, 024429 (2014).

[43] J. Moritz, S. van Dijken, and J. M. D. Coey, Eur. Phys. J. B 45, 191 (2005).

[44] M. Ali, C. H. Marrows, M. Al-Jawad, B. J. Hickey, A. Misra, U. Nowak, and K. D. Usadel, Phys. Rev. B 68, 214420 (2003).

[45] T. Gredig, I. N. Krivorotov, and E. D. Dahlberg, Phys. Rev. B 74, 094431 (2006).

[46] A. Maitre, D. Ledue, and R. Patte, J. Magn. Magn. Mater. 324, 403 (2012).

[47] S. G. E. te Velthuisand A. Berger, G. P. Felcher, B. K. Hill, and E. D. Dahlberg, J. Appl. Phys. 87, 5046 (2000).

[48] J. Lajzerowicz and J. Niez, J. Phys. Lett. 40, 165 (1979). 